\title{
Sacha Inchi (plukenetia volubilis linneo): ¿una experiencia ancestral desaprovechada? Evidencias clínicas asociadas a su consumo
}

\author{
Sacha Inchi (plukenetia volubilis linneo): \\ a wasted ancestral experience? \\ Clinical evidence related to its consumption
}

\begin{abstract}
Sacha Inchi (Plukenetia volubilis L.) is an oleaginous climbing plant that grows in countries like Peru, Bolivia and Colombia. It is an excellent source of unsaturated fatty acids, mainly alpha linolenic (C18:3) and linoleic (C18:2), which represent approximately $82 \%$ of total oil content. Sacha Inchi has been part of the diet of native people of the region since ancient times and is seen as a promising crop today. Due to its composition of essential fatty acids and its high content of antioxidants and tocopherols, the recommendation of its consumption in the diet can be a strategy for the prevention of cardiovascular disease in populations of Latin America, as olive oil consumption known for its cardioprotective properties, is typically low in these populations. The aim of this article is to review the published literature regarding the safety, acceptability and health impact of Sacha Inchi oil.

Key words: Sacha Inchi; Plukenetia volubilis L; fatty acids; healthy food; cardiovascular risk.
\end{abstract}

\section{INTRODUCCIÓN}

Sacha Inchi (Plukenetia volubilis L.) es una planta generalmente trepadora o liana, catalogada por primera vez en 1753 por el naturalista Linneo en la Amazonía peruana. Su nombre deriva de dos palabras quechuas: 'sacha' que significa silvestre, e 'inchi', cuyo término hace referencia al maní que produce. También es conocida como sacha yuchi, sacha yuchiqui, sacha inchik, maní del monte, maní silvestre, maní del inca, entre otras (1). Su nombre de maní de los incas o, en inglés, inca peanut, responde a las evidencias que muestran que fue cultivada por los incas, hace más de 3000 años (2).

El género Plukenetia pertenece a la familia Euforbiáceas y está integrado por 19 especies. Tiene una distribución pantropical, hallándose 12 especies en sudamérica y centroamérica y las restantes en el viejo mundo, pudiendo existir otras especies actualmente no conocidas (3). En América, su presencia se da principalmente en Perú, Bolivia, Antillas Menores, Surinam, Venezuela, Colombia, Ecuador y Brasil, sitios que cumplen sus exigencias óptimas de crecimiento, que incluyen una altitud entre 30 y 2000 m.s.n.m., clima tropical o sub-tropical, con temperaturas de 10 a $26^{\circ} \mathrm{C}$ y una humedad relativa del $78 \%$ (1).
Alicia Norma Alayón (1)

Isabella Echeverri J. (2)

(1) Grupo de Investigaciones Biomédicas (GIB). Facultad de Ciencias de la Salud, Universidad de San Buenaventura, Cartagena, Colombia. (2) Grupo de Investigación Biomédica, Depto. Ciencias Básicas Médicas. Facultad de Ciencias de la Salud, Universidad ICESI, Cali, Colombia.

Dirigir la correspondencia a: Alicia Norma Alayón Profesor-investigador de Tiempo Completo
Cuarto Callejón de Manga, Urbanización Santa Cruz Casa 3. Cartagena de Indias, Colombia. Teléfono celular: 315-7760526

Dirección de correo electrónico: alinorala@gmail.com

Este trabajo fue recibido el 13 de Octubre de 2015 y aceptado para ser publicado el 9 de Marzo de 2016.

De su semilla se extrae un aceite rico en antioxidantes y ácidos grasos poliinsaturados con elevado potencial agro tecnológico y aplicaciones en las industrias alimentarias humanas y animales, así como cosméticas $(1,2,4,5)$.

Tomando en consideración su composición en ácidos grasos y la evidencia existente, es esperable que la mayor efectividad clínica de Sacha Inchi se asocie con su capacidad para mejorar los perfiles lipídicos y disminuir el riesgo cardiovascular, aunque hay experiencias que sugieren que podría también ser efectivo en tratamientos preventivos y curativos de enfermedades de la piel (6), lo que demuestra la amplia variedad de aplicaciones posibles.

No obstante el desarrollo investigativo sobre la composición química de sus aceites y tortas (7-10), el rendimiento de distintos genotipos y fenotipos $(10,11)$, la mejora en la eficiencia de sus cultivos $(4,13-15)$ y sus capacidades en términos de alternativas de negocio (16) o desarrollo industrial (17), son menos abundantes los estudios que profundizan en aspectos relacionados con el impacto metabólico del consumo dietario de estos productos. El propósito de este artículo es hacer una revisión de la literatura publicada en los últimos 15 años sobre la composición y el consumo dietario de la semilla 
de Sacha Inchi o de su aceite, a efectos de reflexionar sobre las ventajas de su ingesta.

\section{METODOLOGÍA}

Se revisaron las bases de datos Elsevier Scopus, EBSCO y Journal Science Direct y se buscaron artículos publicados en el periodo 2000-2105 utilizando como palabras clave Sacha Inchi, Plukenetia volubilis L., maní del Inca e inca peanut en título, resumen y palabras claves, en revistas arbitradas.

\section{Los ácidos grasos y la salud cardiovascular}

El auge del impacto positivo en la salud cardiovascular derivado de la adopción de dietas como la mediterránea, fue puesto en evidencia por los resultados de estudios multicéntricos extensos como el de PREvención con Dleta MEDiterránea (PREDIMED). En promedio, este tipo de dieta provee entre 33 y $42 \%$ de la ingesta calórica total de los individuos en forma de lípidos, $15 \%$ en forma de proteínas y el resto en forma de carbohidratos, lo que la convierte en una dieta relativamente rica en grasa de origen vegetal (18) y otorga un importante papel a los lípidos, implicándolos en los beneficios hallados.

La evidencia actual indica que sus ventajas estarían asociadas con un óptimo balance entre los diferentes alimentos y componentes dietéticos (19) y muy relacionado con el contenido de aceite de oliva $(20,21)$ a lo que se suma su capacidad para evitar la peroxidación de lípidos y de lipoproteínas dado su contenido en tocoferoles, tocotrienoles, $\beta$-caroteno y diferentes compuestos fenólicos con potente acción antioxidante (19).

Desde una perspectiva química, los ácidos grasos pueden clasificarse en saturados e insaturados. Éstos últimos, se reconocen con la letra omega $(\omega)$ seguida por el número correspondiente a la posición del carbono que lleva la insaturación, comenzando a numerar desde el grupo metilo terminal. Entre los ácidos grasos insaturados tenemos los ácidos grasos $\omega$-9 como el ácido oleico (18:1; AO) presente en el aceite de oliva, ácidos grasos $\omega-6$ como el linoleico (18:2; LA) presente en los aceites vegetales y los $\omega-3$ entre los que se encuentra el ácido alfa linolénico (18:3; ALA), que se halla presente en cantidades importantes en algunas especies vegetales como el Sacha Inchi.

El aceite de pescado, rico en ácidos grasos insaturados $\omega-3$, representados fundamentalmente por el ácido eicosapentaenoico (20:5; EPA) y el docosahexaenoico (22:6; DHA) (22), ha mostrado mejorar los perfiles lipídicos, sin embargo la aceptación cultural de su sabor ha dificultado su incorporación a la dieta (23), lo que justifica la necesidad de hallar nuevas fuentes con mejor palatabilidad pero igualmente saludables.

En humanos, las vías metabólicas endógenas para convertir ALA en EPA y EPA en DHA son limitadas (entre 0,2\% a $8 \%$ de ALA es convertido en EPA y entre $0 \%$ y $4 \%$ de ALA a DHA), pero existen estudios que sugieren que ALA podría tener acciones cardiovasculares benéficas, aunque todavía sin resultados concluyentes (24), por lo que se deben continuar estudios del impacto del consumo de fuentes vegetales, ricas en este tipo de ácidos grasos, y evaluar su consideración como sustituto o suplemento en aquellas poblaciones que no consumen los provenientes de fuentes marinas.

\section{Composición de Sacha inchi}

La semilla de Sacha inchi, presenta alrededor de 48-50\% de aceite y $27-28 \%$ de proteínas altamente digeribles y ricas en aminoácidos esenciales, excepto leucina y lisina (2). El aceite obtenido de su semilla está compuesto, en su mayor parte por ácidos grasos poliinsaturados (PUFA) que incluyen 42-48\% de ALA y $32-37 \%$ de LA, con $12 \%$ de monoinsaturados (MUFA). Aun cuando se establecieron diferencias al comparar la composición química de las semillas de diferentes especies, en todas ellas fue apreciable un importante contenido de ALA y una baja relación $\omega-6 / \omega-3$, así como cantidades considerables de tocoferoles, fitosteroles y compuestos fenólicos, responsables del elevado poder antioxidante de sus derivados (25). Semillas de Sacha Inchi cultivada en Colombia, mostraron un elevado contenido de aceite $(41,4 \%)$ y proteínas $(24,7 \%)$, mientras que su aceite, compuesto principalmente de lípidos neutros, estuvo constituido en $50,8 \%$ por ácidos grasos $\omega-3$ y 33,4\% de $\omega-6$, similar a lo hallado en otros países (7).

Al comparar una muestra de aceite obtenida de Sacha inchi cultivado en el Municipio de Ibagué, Colombia, con aceite de oliva virgen, reconocida como un referente saludable para consumo, se encontró que los aceites de Sacha presentaron mayor índice de yodo y menores índices de acidez y de peróxidos, independientemente del método de extracción aplicado (soxhlet o reactor de flujo forzado) lo cual resulta ser una deseable combinación de elevados porcentaje de insaturaciones con adecuados indicadores de estabilidad (26), características congruentes con lo hallado en un aceite de Sacha comercial de Perú (27). El aceite obtenido de semillas de Sacha Inchi de origen peruano, Follegatti y cols. hallaron tocoferoles (gamma y delta) en una concentración de $2,39 \mathrm{~g} / \mathrm{Kg}$, que aportan a conferir resistencia ante procesos de oxidación (28).

Seguridad y ventajas del consumo de Sacha Inchi

A pesar del notable incremento en el número y diversidad de publicaciones con resultados de investigaciones sobre el cultivo de las plantas de Sacha Inchi y la caracterización de sus partes y derivados, no es igualmente abundante las investigaciones orientadas a evaluar cambios metabólicos derivados de su consumo. Sin embargo, aunque menos numerosas, las evidencias actuales muestran un camino prometedor y permiten estimar en las semillas y en los derivados de Sacha Inchi un interesante potencial agroindustrial.

La mayor cantidad de estudios se han desarrollado en Perú, en modelos animales y en humanos, y han permitido demostrar que su consumo es seguro y confiable, lo que también se deduce del hecho de ser un alimento de consumo ancestral. De hecho, numerosas tribus indígenas americanas lo utilizaban para recuperar fuerzas o aprovechaban el uso de su aceite y harina, a manera de ungüento para dolores musculares y con acciones cosméticas $(6,29)$.

Entre los estudios en modelos animales, se destaca una investigación realizada en ratones de la cepa Nish, sometidos a una dieta balanceada y dosis crecientes de aceite de Sacha Inchi, en la cual reportaron una dosis letal DL50 de 111,65 $\mathrm{mL} / \mathrm{kg}$ de peso (30). La mortalidad, en dosis mayor a $64 \mathrm{~mL} /$ $\mathrm{kg}$ peso, fue dosis dependiente y se relacionó con disminución de peso y diarrea.

En ratas macho Holtzman, por su parte, utilizando dosis repetidas por 60 días, se mostró que la ingesta de aceite de Sacha Inchi es inocua en esas condiciones, con una DL50 mayor a $37 \mathrm{~g} / \mathrm{kg}$ de peso corporal, lo cual muestra la seguridad de su consumo en esta especie (31).

Otro estudio en ratas albinas Holtzman, tratadas durante 15 días con una dieta conteniendo $15 \%$ de grasas saturadas, produjo un aumento de colesterol en5,6\%, de triglicéridos en $45 \%$, de VLDL y LDL en 45 y $21 \%$, respectivamente, y disminución de HDL en $25 \%$. A continuación se dividieron en un grupo control negativo que recibió solución fisiológica, 
un grupo control positivo con lovastatina $(20 \mathrm{mg})$ y un grupo intervenido con Sacha Inchi a diferentes dosis. Para los niveles de triglicéridos, los resultados mostraron un descenso del 40,5 $\%$ en el grupo que recibió una dosis de $1 \mathrm{mg} / \mathrm{Kg}$ de peso de Sacha Inchi, y 23 \% en el grupo que recibió lovastatina mientras que en el grupo control negativo se observó un incremento del $1 \%$. No obstante, se vio incremento de triglicéridos en los animales intervenidos con dosis de Sacha de 0,5 y $2 \mathrm{mg} /$ $\mathrm{Kg}$ de peso. Para colesterol de LDL, Sacha inchi mostró ser eficaz a la dosis de 0,5 y $2 \mathrm{ml} / \mathrm{Kg}$ disminuyendo este indicador en $19 \%$ y $7 \%$, respectivamente, con efecto superior al de la lovastatina (6\%). Se observó un incremento de 7,6 y $10 \%$ en los niveles de colesterol total a las dosis de 0,5 y $1 \mathrm{mg} / \mathrm{Kg}$, siendo inferior al aumento en el grupo tratado con lovastatina (14\%). Estos resultados muestran acciones del aceite de Sacha comparables y en ocasiones superiores al tratamiento con lovastatina, y parecen indicar que los efectos sobre la lipemia podrían depender de las dosis, las cuales debieran ser ajustadas convenientemente a fin de optimizar su actividad (30).

En un estudio llevado a cabo en Rattus rattus var albinus alimentadas al inicio con una dieta rica en grasas, se suministró aceite de Sacha inchi, gemfibrozilo o solución fisiológica a fin de comparar el efecto hipolipemiante de los tratamientos. Los resultados mostraron una disminución de los niveles de triglicéridos séricos a la primera y segunda semana de tratamiento, en porcentajes de $35,42 \%$ y $45,57 \%$ respectivamente para Sacha Inchi; $34,81 \%$ y 44,83\% respectivamente para gemfibrozilo y 30,03 \% y 27,24\% respectivamente para solución fisiológica, lo que demuestra una eficacia similar del aceite con respecto al medicamento ensayado (32).

Estudios en personas con hipertrigliceridemia han demostrado que los ácidos grasos $\omega-3$, cuando son consumidos por 12 semanas en concentraciones de 2, 3, y 4 g/día, inducen reducciones de $25,9 \% ; 25,5 \%$ y $30,9 \%$, respectivamente, lo que resulta estadísticamente significativo cuando se compara con la disminución de 4,3\% obtenida con aceite de oliva, siendo efectivo también para la reducción de colesterol no HDL y LDL, mostrando los beneficios de incorporar este tipo de ácidos grasos en el tratamiento de estos desórdenes (33).

De acuerdo con las evidencias disponibles, resulta pertinente enfocar los esfuerzos en el estudio de aceites vegetales ricos en ácidos grasos $\omega-3$, como el caso de Sacha Inchi, tomando en consideración posibles ventajas derivadas de la mejor aceptabilidad del producto, en comparación con los aceites de pescado, y la posibilidad de poder aplicarlo como una alternativa terapéutica dado el menor riesgo de efectos secundarios o interacciones medicamentosas.

En personas aparentemente sanas, el consumo de 10 o $15 \mathrm{~mL}$ de aceite de Sacha Inchi en las mañanas, durante un periodo de 16 semanas, mostró buena aceptabilidad así como ausencia de efectos secundarios clínicos. Muy escasamente se reportó aumento de la frecuencia de las deposiciones, a diferencia de la presencia de diarrea que había sido un efecto hallado en estudios con animales. Lo anterior permitió concluir que el consumo de 10-15 mL de aceite de Sacha Inchi resultaba seguro y aceptable para ser recomendado para consumo humano. En ese mismo estudio se observó una disminución en las cifras de colesterol total y LDL, así como un aumento significativo en el colesterol HDL, sin modificación en los niveles de triglicéridos ni glucosa, aunque con incremento de insulina e índice HOMA, los cuales sin embargo no alcanzaron niveles de insulinoresistencia (34).

En estudios realizados con adultos jóvenes pudo observarse que la adición de 50 g de maní de Sacha Inchi previene el aumento postprandial de triglicéridos producido por la ingesta de una carga de $82 \mathrm{~g}$ de aceite de oliva (35). Sumado a lo anterior, la ingesta de $30 \mathrm{~g}$ de semillas de Sacha Inchi diarios, durante 6 semanas, mostró una notable mejoría de los perfiles lipídicos en el grupo intervenido, comparado con un grupo similar que no consumió Sacha, con disminución en los niveles de colesterol total de 14,02 \%, LDL 20,48 \% y de triglicéridos $36,37 \%$, respecto a los valores iniciales con diferencia significativa respecto a los cambios hallados en el grupo control, que mostró aumentos del 8,15\%; 5,82\% y $6,41 \%$, respectivamente. En el grupo intervenido con Sacha se obtuvo un aumento de $18,97 \%$ en $\mathrm{HDL}$, contra un aumento del $13,1 \%$ en el grupo control (36).

El consumo diario durante 4 meses de 5 o $10 \mathrm{~mL}$ de aceite de Sacha Inchi, conteniendo $2 \mathrm{~g}$ de ácidos grasos $\omega-3$ por cada $5 \mathrm{~mL}$, en pacientes con hipercolesterolemia mostró, para la ingesta de $5 \mathrm{~mL}$, disminución en los niveles de colesterol total, LDL, VLDL, no-HDL, ácidos grasos no esterificados (AGNE) y triglicéridos, aumento significativo de HDL y ausencia de cambios en los niveles de glucosa e insulina; la dosis de $10 \mathrm{~mL}$ mantuvo la disminución en el colesterol total, LDL, no-HDL y AGNE, no mostró disminución de triglicéridos y provocó un ligero incremento de la glucosa e insulina al final del estudio. Lo anterior parece indicar la existencia de una dosis máxima, más allá de la cual la ingesta del aceite no incidiría favorablemente en los marcadores lipídicos de riesgo cardiovascular $y$, para el caso de la insulina, podría verificarse un efecto de saturación en sus receptores. En ningún caso se observaron efectos secundarios ni rechazo a su ingesta, lo que muestra la inocuidad de su consumo y su aceptabilidad (37).

\section{CONCLUSIONES}

El consumo del maní de Sacha Inchi o sus derivados, caracterizados por su elevado contenido de ácidos grasos poliinsaturados $\omega-3$ y $\omega-6$, se ha relacionado con la mejoría del perfil bioquímico relacionado con riesgo cardiovascular, lo que permite suponer beneficios a mediano y largo plazo, en acciones preventivas de distintos niveles.

Su acción, en términos de mejoramiento de los perfiles lipídicos tanto en ayunas como posprandiales, podría resultar una oportunidad sin precedentes de hacer frente, con un producto natural, a los índices de morbimortalidad por estas causas, especialmente tomando en consideración el fracaso de estrategias basadas en la disminución de la ingesta grasa, y en los intentos infructuosos para aumentar las concentraciones de lipoproteínas de alta densidad, que ha sido uno de los hallazgos en las intervenciones con Sacha Inchi.

Sumado a ello, de confirmarse la presencia de actividad biológica comparable a opciones farmacológicas, y una vez ajustadas las dosis que optimicen su actividad, el consumo del aceite o el maní de Sacha podría constituirse en una alternativa natural que evitaría los riesgos de interacciones medicamentosas o efectos secundarios de dichos medicamentos.

Su consumo ancestral, sumado a los resultados de las investigaciones en modelos animales y en humanos, ha demostrado la inocuidad y ha permitido evidenciar que su sabor no es un problema al momento de considerar su inclusión como aceite de mesa, en forma de complemento alimenticio, o como alternativa terapéutica. La presencia de compuestos antioxidantes podrían contribuir con efectos protectores del daño oxidativo celular, al tiempo que otorgan al producto características fisicoquímicas que aseguran su estabilidad y le confieren un elevado potencial agroindustrial.

Es importante continuar los estudios que permitan 
revalorizar esta fuente vegetal de ácidos grasos, desde una doble dimensión, lo que corresponde al ámbito de la salud cardiovascular de los consumidores, y desde una mirada agroindustrial de país que incluye a todos los estamentos involucrados en su cultivo, industrialización y comercialización, viéndola además como una oportunidad de cultivo alternativo y una opción válida para el desarrollo de nuestras comunidades campesinas.

\section{RESUMEN}

Sacha Inchi (Plukenetia volubilis L.) es una planta oleaginosa trepadora que crece en países como Perú, Bolivia y Colombia. Es una excelente fuente de ácidos grasos insaturados, fundamentalmente alfa linolénico (C18:3) y linoleico (C18:2), que representan aproximadamente $82 \%$ del contenido total del aceite. Sacha Inchi ha sido parte de la dieta de indígenas desde la antigüedad y es visto en la actualidad como un cultivo prometedor. Por su composición en ácidos grasos esenciales y su alto contenido en tocoferoles y antioxidantes, la recomendación de su consumo en la dieta habitual puede ser una estrategia encaminada a la prevención de enfermedades cardiovasculares en poblaciones de América Latina, en las que el consumo de aceite de oliva, reconocido por sus propiedades cardioprotectoras, es bajo. Este artículo tiene por objetivo hacer una revisión de la literatura publicada en relación con la inocuidad, aceptabilidad e impacto sobre la salud, del aceite de Sacha Inchi.

Palabras clave: Sacha Inchi; Plukenetia volubilis L; ácidos grasos; alimento saludable; riesgo cardiovascular.

\section{BIBLIOGRAFÍA}

1. Arfini F, Antonioli F. Sacha inchi. Research about the conditions for recognition of geographical indications in Peru, CRED, Lima Peru, 2013.

2. Flores $D$, Lock $O$. Reassessing the ancient use of Sacha inchi (Plukenetia volubilis L) for nutrition, health and cosmetics. Rev Fitoterapia 2013; 13(1): 23-30.

3. Dostert N, Grischa JR, Cano A, La Torre MI, Weigend M. Factsheet: Botanic information of Sacha inchi Plukenetia volubilis L. Lima Perú, 2009.

4. Viegas PH, Bordignon SR, Bovi G. Horticultural performance of in vitro propagated plants of Sacha inchi. Ciência Rural, Santa Maria 2014; 44(6):1050-1053.

5. Lucas JL, Icochea $E$, Valdivia $R$, Carcelén F, Guzmán J. Effect of Sacha inchi oil (Plukenetia volubilis) in the diet of broiler Breeders on the productive performance of their progeny. Rev Inv Vet Perú 2011; 22 (4):283-9.

6. Gonzalez G, Belkhelfa H, Haddioui L, Bourdy G, Deharo E. Sacha Inchi Oil (Plukenetia volubilis L.), effect on adherence of Staphylococus aureus to human skin explant and keratinocytes in vitro. J Ethnopharmacol 2015; 171:330-4.

7. Gutiérrez LF, Rosada LM, Jiménez A. Chemical composition of Sacha Inchi (Plukenetia volubilis L.) seeds and characteristics of their lipid fraction, Grasas y Aceites 2011; 62(1):76-83.

8. Niu L, Li J, Chen MS, Xu ZF. Determination of oil contents in Sacha inchi (Plukenetia volubilis) seeds at different developmental stages by two methods: Soxhlet extraction and time-domain nuclear magnetic resonance. Industrial Crops Products 2014; 56:187-90.

9. Pereira AH, Gohara AK, Rodrigues AC, Souza NE, Visentaine JV, Matsushita M. Sacha inchi as potential source of essential fatty acids and tocopherols: multivariate study of nut and shell, Acta Scientiarum. Technol Maringá 2013;
35(4):757-63.

10. Betancourth CF. Use of the Sacha inchi residual cake (Plukenetia volubilis L.) by solvent extraction of its oil. Master's of Sustainable Development and Environment. University of Manizales. Colombia, 2013.

11. Wang $X, X u R$, Ruling Wang $R$, Liu A. Transcriptome analysis of Sacha Inchi (Plukenetia volubilis L.) seeds at two developmental stages, BMC Genomics 2012; 13:716.

12. Wang $X$, Liu A. Expression of genes controlling unsaturated fatty acids biosynthesis and oil deposition in developing seeds of Sacha inchi (Plukenetia volúbilis L.), Lipids 2014; 49:1019-31.

13. Cai ZQ. Shade delayed flowering and decreased photosynthesis, growth and yield of Sacha inchi (Plukenetia volubilis) plants. Industrial Crops Products 2011; 34:1235-37.

14. La Rosa R, Quijada J. Germination of Sacha inchi, Plukenetia volubilis L. (Mcbride, 1951) (Malpighiales, Euphorbiaceae) under four different conditions. Biologist (Lima) 2013; 11(1): 9-14.

15. Ruiz H, Mesén F. Effect of 4 doses of Indole butyric acid and types of cutting on the rooting of sacha inchi (Plukenetia volubilis L.). Agronomía Costarricense 2010; 34(2): 259-67.

16. Chirinos $O$, Adachi L, Calderón F, Díaz R, Larrea L, Mucha $G$, Roque L. Sacha inchi oil exports to U.S. market. Lima: ESAN University, 2009.

17. Kumar B, Smita K, Cumbal L, Debut A. Sacha inchi (Plukenetia volubilis L.) oil for one pot synthesis of silvernanocatalyst: An ecofriendly approach Industrial, Crops Products 2014; 58:238-43.

18. Márquez $F$, Bulló $M$, Vizmanos $B$, Casas $P$, Salas J. A pattern of healthy feeding: the traditional Mediterranean diet, Antropo. 2008; 16:11-22.

19. Carbajal A, Ortega R. Mediterranean diets as an example of a prudent and healthy diet. Rev Chil Nutr. 2001; 28(2):224-36.

20. Guasch $M$, Hu FB, Martínez MA, Fitó $M$, Bulló $M$, Estruch $R, \operatorname{Ros} E$, et al. Olive oil intake and risk of cardiovascular disease and mortality in the PREDIMED Study, BMC Med. 2014; $12: 78$.

21. Estruch R, Ros E, Salas-Salvadó J, Covas MI, Corella D, Arós F, Gómez-Gracia E et al. Primary Prevention of Cardiovascular Disease with a Mediterranean Diet. New England J Med 2013; 368(14):1279-90.

22. Nasiff A, Meriño E. Omega-3 fatty acid: blue fish meat and fish oil concentrates. The good and the bad. Rev Cubana Med. 2003; 42(2): 49-55.

23. Yaxley A, Miller M, Fraser RJ, Cobiac L, Crotty M. Testing the acceptability of liquid fish oil in older adults, Asia Pac J Clin Nutr 2011; 20 (2): 175-179.

24. Mozaffarian D, Wu JHY. Omega-3 Fatty Acids and Cardiovascular Disease Effects on Risk Factors, Molecular Pathways, and Clinical Events, JACC 2011; 58(20):2047-67.

25. Chirinos $R$, Zuloeta $G$, Pedreschi $R$, Mignolet $E$, Larondelle $Y$, Campos D. Sacha inchi (Plukenetia volubilis): A seed source of polyunsaturated fatty acids, tocopherols, phytosterols, phenolic compounds and antioxidant capacity, Food Chem 2013; 141:1732-9.

26. Castaño DL, Valencia MP, Murillo E, Mendez JJ, Joli JE. Fatty acid composition of Inca peanut (Plukenetia volúbilis linneo) and its relationship with vegetal bioactivity. Rev Chil Nutr. 2012; 39(1):45-52.

27. Vicente J, de Carvalho MG, Garcia-Rojas EE, Fatty acids profile of Sacha inchi oil and blends by 1 H NMR and GC- 
FID, Food Chem. 2015;181: 215-21.

28. Follegatti LA, Piantino CR, Grimaldi $R$, Cabral FA. Supercritical $\mathrm{CO} 2$ extraction of omega-3 rich oil from Sacha inchi (Plukenetia volubilis L.) seeds, J. Supercritical Fluids 2009; 49:323-9.

29. Mosquera T, Noriega P, Tapia W, Pérez SH. Effectiveness evaluation of cosmetic creams elaborated from oils extracted from amazon plants: Mauritia flexuosa (morete), Plukenetia volubilis (sacha inchi) and Oenocarpus bataua (ungurahua). Rev Ciencias Vida 2012; 16(2):14-22.

30. Cordova M, Cotillo J, Cruz S, Espinoza D, Felix L, Fernán$\operatorname{dez} D$, et al. Evaluation of acute toxiclty and hypollpemic action of Plukenetia volúbilis, Sacha inchi. Rev Horizonte Méd 2006; 6(1):45-52.

31. Gorriti A, Arroyo J, Quispe F, Cisneros B, Condorhuama M, Almora Y, Chumpitaz V. Oral toxicity at 60-days of Sacha inchi oil (Plukenetia volubilis L.) and linseed (Linum usitatissimum I.), and determination of lethal dose 50 in rodents. Rev Peru Med Exp Salud Pública 2010; 27(3): 352-60.

32. Vicuña A, Izquierdo EJ, Huamán JJ. Gemfibrozil versus Sacha Inchi oil in reducing serum triglyceride levels in Rattus rattus var albinus. Acta Méd Peruana 2012; 29 (2):85-8.
33. Kastelein JJ, Maki KC, Susekov A, Ezhov M, Nordestgaard $B G$, et al. Omega-3 free fatty acids for the treatment of severe hypertriglyceridemia: The EpanoVa fOr Lowering Very high triglyceridEs (EVOLVE) trial. J Clin Lipidol. 2014; 8: 94-106.

34. Gonzales GF, Gonzales C. A randomized, double-blind placebo-controlled study on acceptability, safety and efficacy of oral administration of sacha inchi oil (Plukenetia volubilis L.) in adult human subjects, Food Chem Toxicol. 2014; 65:168-76.

35. Huamán J, Chávez $K$, Castañeda E, Carranza S, Chávez T, et al. Effect of Plukenetia volubilis Linneo (Sacha inchi) on postprandial triglycerides. An Fac Med. 2008; 69(4): 263-6.

36. Huamán JJ, Fogel BE; Escobar, PI, Castillo KY. (2012). Effects of the ingestión of Plukenetia volubilis Linneo (a.k.a. 'Sacha inchi') on the lipid profile of young adults. Acta Méd Peruana 2012; 29(3):155-60.

37. Garmendia F, Pando R, Ronceros G. Effect of Sacha inchi oil (Plukenetia volubilis I) on the lipid profile of patients with hyperlipoproteinemia. Rev Peru Med Exp Salud Pública $2011 ; 28(4): 628-32$. 